\title{
Norms of Elementary Operators
}

\author{
RICHARD M. TIMONEY
}

\begin{abstract}
We make some simple remarks about the norm problem for elementary operators in the contexts of algebras of operators on Banach spaces and $C^{*}$-algebras. We illustrate a lack of symmetry in the problem for algebras containing the finite rank operators and also for the Calkin algebra. An isomorphic symmetry is shown for subhomogeneous $C^{*}$ algebras.
\end{abstract}

For $A$ an algebra, an operator $T: A \rightarrow A$ is called an elementary operator if $T$ can be expressed in the form

$$
T a=\sum_{i=1}^{\ell} a_{i} a b_{i}
$$

with $a_{i}$ and $b_{i}(1 \leq i \leq \ell)$ in $A$. For $A$ a $C^{*}$-algebra, one may allow $a_{i}$ and $b_{i}$ to be in the multiplier algebra of $A$ (see [3]). Such representations of $T$ may not be unique. We write $\mathcal{E}(A)$ for the class of elementary operators on $A$. We will always be considering normed algebras $A$ and so there is a natural operator norm on $\mathcal{E}(A)$ (which is contained in the bounded linear operators on $A$ ).

The so-called norm problem (see [5] for a survey) asks for $\|T\|$ to be expressed in terms of $\left\|a_{i}\right\|$ and $\left\|b_{i}\right\|$ (or, more realistically, in terms of the norms of some combination of the $a_{i}$ and some combination of the $b_{i}$ such as in the Haagerup norm mentioned below). In the case $\ell=1$ and $T a=a_{1} a b_{1}$, we know from [4] that if $A$ is ultraprime then there is a constant $c_{A}>0$ such that $c_{A}\left\|a_{1}\right\|\left\|b_{1}\right\| \leq\|T\|$. In fact, we can take the existence of $c_{A}$ as the definition of $A$ being ultraprime. Of course $\|T\| \leq\left\|a_{1}\right\|\left\|b_{1}\right\|$ for any normed algebra $A$.

For $A$ ultraprime, the map from the algebraic tensor product $A \otimes A$ to $\mathcal{E}(A)$ which maps $\sum_{j=1}^{\ell} a_{j} \otimes b_{j}$ to $T \in \mathcal{E} \ell(A)$ of the form (1) is bijective (see [4, Theorem 5.1]). Hence we can define $T^{f} \in \mathcal{E} \ell(A)$ (for $T \in \mathcal{E}(A)$ as in (1)) by $T^{f} a=\sum_{i=1}^{\ell} b_{i} a a_{i}$. 
In a recent preprint $\mathrm{V}$. Runde [8] considers algebras $A$ of operators on infinite dimensional reflexive Banach spaces $E$ with the approximation property. If $A$ contains the finite rank operators $\mathcal{F}(E)$, he has shown that the map $T \mapsto T^{f}$ is not norm continuous on $\mathcal{E}(A)$. Following [8], we refer to $T \mapsto T^{f}$ as the flip map on $\mathcal{E}(A)$.

Note that algebras $A$ with $\mathcal{F}(E) \subseteq A \subseteq \mathcal{B}(E)$ (= the bounded operators on a Banach space $E$ ) are always ultraprime. Our main result is an elementary proof of the following variant of the result of Runde [8].

Theorem 1. Assume that $E$ is a Banach space that contains an infinite dimensional complemented subspace with a Schauder basis, and suppose $A$ is an algebra of bounded operators on $E$ with $\mathcal{F}(E) \subseteq$ A. (We give $A$ the operator norm.) Then the map $T \mapsto T^{f}$ is not norm continuous on $\mathcal{E}(A)$.

Proof. Let $\left(e_{n}\right)_{n=1}^{\infty}$ be a basic sequence in $E$ with closed linear span complemented in $E$ via a projection $P$. Then the projections $\pi_{n}\left(\sum_{j=1}^{\infty} x_{j} e_{j}\right)=\sum_{j=1}^{n} x_{j} e_{j}$ are uniformly bounded on the span of the $e_{j}$ and so $P_{n}=\pi_{n} \circ P$ are uniformly bounded on $E$, and belong to $\mathcal{F}(E)$.

Consider the biorthogonal functionals $f_{j}$ to the $e_{n}$ (on the span of the $\left.e_{n}\right)$ given by $f_{j}\left(\sum_{n=1}^{\infty} x_{n} e_{n}\right)=x_{j}$ and then $F_{j}=f_{j} \circ P$. Define $E_{i j} \in \mathcal{F}(E)$ by $E_{i j}(x)=F_{j}(x) e_{i}$. Consider $T_{n} \in \mathcal{E} \ell(A)$ given by

$$
T_{n} a=\sum_{i=1}^{n} E_{i 1} a E_{1 i} \quad(a \in A) .
$$

For $x \in E$, we have

$$
\begin{aligned}
\left(T_{n} a\right)(x) & =\sum_{i=1}^{n} E_{i 1}\left(a\left(E_{1 i}(x)\right)\right) \\
& =\sum_{i=1}^{n} E_{i 1}\left(a\left(F_{i}(x) e_{1}\right)\right)=\sum_{i=1}^{n} F_{i}(x) E_{i 1}\left(a\left(e_{1}\right)\right) \\
& =\sum_{i=1}^{n} F_{i}(x) F_{1}\left(a\left(e_{1}\right)\right) e_{i}=F_{1}\left(a e_{1}\right) \sum_{i=1}^{n} F_{i}(x) e_{i} \\
& =F_{1}\left(a e_{1}\right) P_{n}(x)
\end{aligned}
$$

Thus $\left\|T_{n} a\right\| \leq\left\|F_{1}\right\|\left\|e_{1}\right\|\left\|P_{n}\right\|\|a\|$ or $\left\|T_{n}\right\| \leq\left\|F_{1}\right\|\left\|e_{1}\right\|\left\|P_{n}\right\|$ and consequently $\sup _{n}\left\|T_{n}\right\|<\infty$. 
Working with $T_{n}^{f}$, we get from a similar calculation

$$
\left(T_{n}^{f} a\right)(x)=\sum_{i=1}^{n} E_{1 i}\left(a\left(E_{i 1}(x)\right)\right)=F_{1}(x) \sum_{i=1}^{n} F_{i}\left(a\left(e_{i}\right)\right) e_{1}
$$

Taking $a=P_{n}$, we have $\left(T_{n}^{f} P_{n}\right)(x)=F_{1}(x) \sum_{i=1}^{n} e_{1}=n F_{1}(x) e_{1}$. Thus $\left\|T_{n}^{f} P_{n}\right\|=n\left\|F_{1}\right\|\left\|e_{1}\right\| \rightarrow \infty$ as $n \rightarrow \infty$. As $\sup _{n}\left\|P_{n}\right\|<\infty$, it follows that $\left\|T_{n}^{f}\right\| \rightarrow \infty$ as $n \rightarrow \infty$.

Remark 1. We consider now the case where $E=\ell^{2}$ is a (separable) Hilbert space and we assume that $A$ is a $C^{*}$-algebra of operators on $\ell^{2}$. We are then restricted to the two examples $A=\mathcal{K}\left(\ell^{2}\right)=$ the compact operators and $A=\mathcal{B}\left(\ell^{2}\right)$, if $A$ is to contain all the finite rank operators (as is required to apply the theorem).

We could also consider the completely bounded norm of operators $T \in \mathcal{E} \ell(A)$. For $T \in \mathcal{E} \ell(A)$ given by (1), the completely bounded norm is $\|T\|_{c b}=\inf \sqrt{\left\|\sum_{j=1}^{\ell} a_{j} a_{j}^{*}\right\|\left\|\sum_{k=1}^{\ell} b_{k}^{*} b_{k}\right\|}$, where the infimum is taken over all possible representations of $T$. In fact this is a wellknown relation between $\|T\|_{c b}$ and the Haagerup norm on the tensor product $A \otimes A$ for these examples (see $[2,9]$ ). The $T_{n}$ constructed as above from an orthonormal basis $\left(e_{i}\right)_{i=1}^{\infty}$ have $\sup _{n}\left\|T_{n}\right\|_{c b}<\infty$ while $\left\|T_{n}^{f}\right\|$, and hence $\left\|T_{n}^{f}\right\|_{c b}$, is unbounded.

Further, $T_{n}$ is actually completely positive (see [6] for the definition) and we could even modify $T_{n}$ to be unital by adding a term $\left(\mathrm{id}-\pi_{n}\right) a\left(\mathrm{id}-\pi_{n}\right)$.

Remark 2. In the case of general $C^{*}$-algebras, there are results on elementary operators where subhomogeneous $C^{*}$-algebras behave as though they were finite dimensional. At the opposite extreme, antiliminal $C^{*}$-algebras sometimes have the same behaviour. (See for example $[1,10]$.)

Subhomogeneous $C^{*}$-algebras are those where there is a finite bound on the dimensions of irreducible $*$-representations. Write $A$ for the algebra and $\hat{A}$ for the class of all inequivalent irreducible representations $\pi: A \rightarrow \mathcal{B}\left(H_{\pi}\right)$ of $A$. Then $A$ subhomogeneous means that $\sup _{\pi \in \hat{A}} \operatorname{dim} H_{\pi}=k<\infty$. (We call $A k$-subhomogeneous when we want to refer to an upper bound for the dimensions.) Abelian $A$ are the 1-subhomogeneous $C^{*}$-algebras and of course then the flip map is the identity on $\mathcal{E} \ell(A)$. 
The atomic representation of $A$ (see $[7,4.3 .7])$ is given by

$$
\pi_{a}: A \rightarrow \bigoplus_{\pi \in \hat{A}} \mathcal{B}\left(H_{\pi}\right): a \mapsto(\pi(a))_{\pi \in \hat{A}}
$$

Here $\bigoplus_{\pi \in \hat{A}} \mathcal{B}\left(H_{\pi}\right)$ means the $\ell^{\infty}$ sum and it is a dual $C^{*}$-algebra in a natural way. Hence it is a von Neumann or $W^{*}$-algebra. The range of $\pi_{a}$ is weak*-dense in $\mathcal{A}=\bigoplus_{\pi \in \hat{A}} \mathcal{B}\left(H_{\pi}\right)$, and there is a simple way to extend any $T \in \mathcal{E}(A)$ to an elementary operator on $\mathcal{A}$. This extension is the unique weak*-continuous $T_{a}: \mathcal{A} \rightarrow \mathcal{A}$ satisfying $T_{a} \circ \pi_{a}=\pi_{a} \circ T$.

From this perspective, one can see that an elementary operator $T \in \mathcal{E}(A)$ given by (1) is zero if and only if

$$
\sum_{j=1}^{\ell} \pi\left(a_{j}\right) \otimes \pi\left(b_{j}\right)=0 \in \mathcal{B}\left(H_{\pi}\right) \otimes \mathcal{B}\left(H_{\pi}\right)
$$

for each $\pi \in \hat{A}$. From this we can deduce that $T^{f}$ is well-defined (independent of the representation (1) of $T$ ) when $A$ is a $C^{*}$-algebra, even though $A$ may not be ultraprime.

We can pass to $T_{a}$ from $T \in \mathcal{E} \ell(A)$ of the form (1) and note that $\left(T^{f}\right)_{a}=\left(T_{a}\right)^{f}$. Also $\|T\|$ is the supremum over $\pi \in \hat{A}$ of the norms of the elementary operators

$$
x \in \mathcal{B}\left(H_{\pi}\right) \mapsto \sum_{j=1}^{\ell} \pi\left(a_{j}\right) x \pi\left(b_{j}\right) .
$$

We can apply finite-dimensional estimates (depending on the dimension) to deduce that $\left\|T^{f}\right\| \leq C_{k}\|T\|$ when $A$ is $k$-subhomogeneous.

The simplest example of an antiliminal $C^{*}$-algebra is the so-called Calkin algebra $A=\mathcal{B}\left(\ell^{2}\right) / \mathcal{K}\left(\ell^{2}\right)$. Our examples $T_{n}$ are built using finite rank $a_{i}$ and $b_{i}$ and so they vanish when quotiented by the compact operators. However, we can replace them with rather similar infinite multiplicity examples that will survive the quotient map. One way to do that is to note that $\ell^{2}$ is unitarily equivalent to the Hilbert space tensor product $\ell^{2} \otimes_{2} \ell^{2}$. We can take

$$
S_{n} a=\sum_{i=1}^{n}\left(E_{i 1} \otimes \operatorname{id}_{\ell^{2}}\right) a\left(E_{1 i} \otimes \mathrm{id}_{\ell^{2}}\right)
$$

and show via a similar computation to that used for $T_{n}$ that even on the Calkin algebra, $\sup _{n}\left\|S_{n}\right\|_{c b}<\infty$ while $\left\|S_{n}^{f}\right\|$ is unbounded. 
Question 1. For which Banach spaces $E$ does the conclusion of Theorem 1 hold for all algebras of operators on $E$ that contain the finite ranks?

Theorem 1 covers quite a few common spaces, but not all $E$ covered by the result of [8]. Moreover $\ell^{\infty}$ fails to satisfy the hypotheses of Theorem 1, but the proof can be carried through in that case.

Question 2. Is the flip $T \mapsto T^{f}$ unbounded on $\mathcal{E}(A)$ for all nonsubhomogeneous $C^{*}$-algebras $A$ ?

\section{REFERENCES}

[1] R. J. Archbold, M. Mathieu and D. W. B. Somerset, Elementary operators on antiliminal $C^{*}$-algebras, Math. Ann. 313 (1999), 609-616.

[2] U. Haagerup, The $\alpha$-tensor product of $C^{*}$-algebras, unpublished manuscript, Univ. of Odense (1980).

[3] M. Mathieu, Elementary operators on prime $C^{*}$-algebras. I, Math. Ann. 284 (1989) 223-244.

[4] M. Mathieu, Rings of quotients of ultraprime Banach algebras. With applications to elementary operators, in R. J. Loy (ed), 'Conference on Automatic Continuity and Banach Algebras', Australian National University (1989) pp. 297-317.

[5] M. Mathieu, The norm problem for elementary operators, in Recent Progress in Functional Analysis, K. D. Bierstedt et al (eds.), North-Holland Math. Studies, Elsevier, Amsterdam, 2001, in press.

[6] V. I. Paulsen, Completely bounded maps and dilations, Pitman Notes in Mathematics Series Vol. 146, Pitman, New York, 1986.

[7] G. K. Pedersen, $C^{*}$-algebras and their automorphism groups, Academic Press, London, 1979

[8] V. Runde, The flip is often discontinuous, J. Operator Th., to appear.

[9] R. R. Smith, Completely bounded module maps and the Haagerup tensor product, J. Funct. Anal. 102 (1991) 156-175.

[10] R. M. Timoney, An internal characterisation of complete positivity for elementary operators, Proc. Edinburgh Math. Soc., to appear.

Richard M. Timoney,

School of Mathematics,

Trinity College,

Dublin 2, Ireland

richardt@maths.tcd.ie

Received on 28 November 2000 and in revised form on 5 June 2001.

Editorial Note. An argument similar to Theorem 1 for the case of Hilbert space was provided by A. W. Wickstead (private communication). 